\title{
Tolerability, Safety, and Effectiveness of Oxycodone DETERx in Elderly Patients $\geq 65$ Years of Age with Chronic Low Back Pain: A Randomized Controlled Trial
}

\author{
Ernest A. Kopecky ${ }^{1} \cdot$ Ben Vaughn $^{4} \cdot$ Scott Lagasse $^{2} \cdot$ Melinda O'Connor $^{3}$
}

Published online: 10 June 2017

(c) The Author(s) 2017. This article is an open access publication

\begin{abstract}
Trial Design This was a phase III, randomized withdrawal, double-blind, placebo-controlled, enriched enrollment, parallel-group, multicenter study intended to demonstrate the safety, tolerability, and analgesic efficacy of oxycodone DETERx $^{\circledR}\left(\right.$ Xtampza $^{\mathrm{TM}}$ ER) compared with matching placebo.

Methods This post hoc analysis was performed using data from a subpopulation of enrolled patients who were $\geq 65$ years of age. The study enrolled male and female patients with a clinical diagnosis of moderate-to-severe chronic low back pain for a minimum of 6 months prior to screening who required around-the-clock opioid therapy. To be eligible for enrollment, patients were required to have an average 24-h pain intensity score of $\geq 5$ and $\leq 9$ on an 11-point (0-10) Pain Intensity-Numerical Rating Scale at the screening visit. The study enrolled both opioid-experienced and opioid-naïve patients. The study consisted of an open-label titration phase followed by a 12 -week double-blind maintenance phase. The dose range was 40-160 mg oxycodone hydrochloride equivalent per day. This post hoc analysis evaluated the safety, tolerability, and effectiveness of oxycodone DETERx among patients
\end{abstract}

Melinda O'Connor

moconnor@collegiumpharma.com

1 Clinical Development, Collegium Pharmaceutical, Inc., 780 Dedham Street, Suite 800, Canton, MA 02021, USA

2 Medical Affairs, Collegium Pharmaceutical, Inc., 780 Dedham Street, Suite 800, Canton, MA 02021, USA

3 Clinical Operations, Collegium Pharmaceutical, Inc., 780 Dedham Street, Suite 800, Canton, MA 02021, USA

4 Biostatistics, Rho, Inc., 6330 Quadrangle Drive, Chapel Hill, NC 27517, USA $\geq 65$ years of age. The effectiveness of oxycodone DETERx was evaluated based on average pain intensity scores, Patient Global Impression of Change, responder analysis, and Kaplan-Meier survival analysis. The safety and tolerability of oxycodone DETERx were also evaluated. Patients were randomized to either oxycodone DETERx or placebo using a blocked randomization scheme in a 1:1 ratio. Randomization was stratified by previous opioid use (naïve or experienced). The study drug was coded in a manner that maintained the blinding. Study personnel and patients remained blinded to the assigned treatments throughout the study.

Results For this post-hoc analysis, the intent-to-treat and randomized safety populations included 52 patients $\geq 65$ years old, 26 each in the oxycodone DETERx and placebo groups, who participated in the study during the titration phase and were randomized to the double-blind maintenance phase. Clinically important pain reduction from screening was achieved with oxycodone DETERx, with the median pain intensity score decreasing from 7.50 at screening to 2.69 at Week 12 . A clinically meaningful treatment difference of -0.9 in pain score between oxycodone DETERx and placebo was observed. All 18 elderly patients who completed the study reported improvement in pain, with $62 \%$ showing $\geq 30 \%$ improvement and $54 \%$ showing $\geq 50 \%$ improvement in pain intensity compared with patients on placebo $(p=0.0128$ and $p=0.0501$, respectively). Patients on oxycodone DETERx remained in the study longer than those on placebo. Of the 26 patients $\geq 65$ years old randomized to continue oxycodone DETERx during the double-blind maintenance phase, 18 (69\%) completed the study; only two patients $(8 \%)$ in the oxycodone DETERx group discontinued due to adverse events. The safety and tolerability profiles showed no new or unexpected safety concerns. The adverse event profiles 
were similar between the titration and double-blind maintenance phases.

Conclusions Oxycodone DETERx was efficacious and generally well tolerated in patients $\geq 65$ years old.

Trial Registration The study was registered with ClinicalTrials.gov (NCT01685684).

\section{Key Points}

Oxycodone DETERx is an easy-to-administer, abuse-deterrent alternative to hard, monolithic tablet formulations of extended-release opioids that may be difficult to swallow, particularly in the elderly.

This analysis showed that oxycodone DETERx was generally efficacious in patients $\geq 65$ years old.

This analysis showed that oxycodone DETERx was well tolerated in the $\geq 65$-year-old subgroup of the study population, with no newly identified (i.e., adverse events currently not already known based on the pharmacology of opioids) safety concerns.

\section{Introduction}

Chronic pain is a global health problem estimated to affect approximately 1.5 billion people worldwide [1]. It increasingly affects the elderly as the incidence of chronic pain increases with age. The elderly population in the US is increasing faster than the total population, according to the 2010 US census [2]. Managing chronic pain in this growing patient population is a public health concern.

Chronic pain is often treated with opioid medications, which can provide short- or long-acting analgesia, depending on the specific properties of the analgesic and its formulation [3]. Long-acting/extended-release (ER) opioids are typically used for around-the-clock maintenance treatment.

Escalating medical use of opioids has been correlated with an increase in the incidence of non-medical opioidrelated drug abuse and manipulation of conventional ER dosage forms for abuse [4]. In an effort to mitigate this type of product manipulation, abuse-deterrent formulations have been developed, including harder tablet formulations that are more difficult to crush. An unintended consequence of hard, monolithic-tablet opioid formulations is that the tablets can be difficult to swallow for some patients, especially the elderly. It is estimated that over 11 million patients in the US with chronic pain either have difficulty swallowing tablets (dysphagia) or dislike swallowing tablets; the Food and Drug Administration (FDA) estimated that as many as $40 \%$ of Americans may suffer from dysphagia $[5,6]$.

Despite warnings in the prescribing information and Patient Medication Guides, patients with dysphagia and their caregivers often manipulate currently marketed ER opioid analgesics by chewing, cutting, or crushing tablets, or opening ER capsules to make swallowing easier [5, 7]. This unintentional or possibly intentional misuse of opioids can cause a change in the release profile of the analgesic, leading to adverse effects, including death. Specifically, altering an ER formulation can change the pharmacokinetic (PK) characteristics (increased maximum plasma concentration $\left[C_{\max }\right]$ and possibly area under the plasma concentration-time curve [AUC]) and can lead to overdose, potentially resulting in death.

Currently available treatment options for patients with chronic pain who cannot swallow solid oral dosage forms include transdermal patches, immediate-release opioids, methadone, and certain other ER opioids, each of which have limitations that can potentially result in inadequate pain management [8-11]. For example, transdermal patches may have unreliable absorption, particularly in the elderly, and are associated with dermal side effects such as skin rash and erythema. Release kinetics can be altered by changes in temperature or use of occlusive dressings [8-10]. Most of the currently available ER opioids do not offer multiple modes of administration (e.g., the same opioid that can be taken via a method other than swallowing intact) or have restrictions for dosing (e.g., take with sufficient water to avoid getting stuck in the throat or do not chew to avoid a potentially fatal overdose).

Oxycodone DETERx ${ }^{\circledR}{ }^{\left(X_{1}\right.}$ Xmpza $^{\mathrm{TM}}$ ER, Collegium Pharmaceutical, Inc., Canton, MA, USA) addresses the needs of patients with chronic pain with dysphagia. This novel, abuse-deterrent formulation is a microsphere-incapsule formulation that enables patients with dysphagia to open the capsule and administer the contents onto soft food, directly into the mouth from a cup, or directly into an enteral tube, while maintaining the ER properties of the product $[12,13]$. The formulation is designed to protect against bolus release and subsequent exacerbation of potentially serious adverse effects if a patient chews or crushes the formulation [12]. The formulation also allows for administration through enteral tubes (e.g., nasogastric and gastrostomy) without the concern for tube blockage or interaction with common administration media such as tube feeding formulas, water, or milk [12-14].

In a phase III study conducted by Collegium Pharmaceuticals, Inc., oxycodone DETERx was found to be safe and efficacious in the management of moderate-to-severe chronic low back pain (CLBP) [3]. 
Because limited data exist on the effects of oxycodone treatment regimens among elderly patients with chronic pain, a post hoc analysis was performed using data for patients $\geq 65$ years of age who participated in the phase III study; the current manuscript reports the safety, tolerability, and effectiveness of oxycodone DETERx in this patient population.

\section{Materials and Methods}

Post hoc analyses were performed using data from a double-blind, placebo-controlled, enriched enrollment, randomized withdrawal study with a titration phase followed by a 12 -week double-blind maintenance phase, which has been described previously [15]. The study was registered with ClinicalTrials.gov (NCT01685684).

\subsection{Patient Population}

The study enrolled opioid-experienced and opioid-naïve male and female patients aged 18-75 years with a clinical diagnosis of moderate-to-severe CLBP for a minimum of 6 months. Eligible patients required around-the-clock opioid therapy and had an average 24-h pain intensity score of $\geq 5$ and $\leq 9$ (i.e., moderate-to-severe pain) on an 11-point (0-10) Pain Intensity-Numerical Rating Scale (PI-NRS) at screening while on their current opioid or non-opioid analgesic medication. Pain was classified as non-malignant and non-neuropathic (Class 1 and 2), neuropathic (Class 3), or symptomatic for more than 6 months after low back pain surgery (Class 9) based on Quebec Task Force Scale score. Patients must have been in otherwise general good health, which was defined as the absence of any clinically relevant abnormalities, based on screening physical examination, medical history, 12-lead electrocardiogram, and clinical laboratory values (hematology, chemistry, serology, and urinalysis). For opioid-naïve patients, each patient must have failed prior acetaminophen (APAP) and/or nonsteroidal anti-inflammatory drug (NSAID) therapy and/or was unable to tolerate NSAIDs. All patients had to have stopped use of all analgesics other than study drug from the day of the first titration phase visit through to end of study or early discontinuation. Adjunct therapy for back pain such as physical therapy, biofeedback therapy, transcutaneous electrical nerve stimulation, acupuncture, nutraceuticals, herbal remedies, and water aerobics must have been kept unchanged for at least 4 weeks prior to screening and through to the end of study/early discontinuation.

To be eligible for the double-blind maintenance phase, patients must have attained a stable dose of oxycodone DETERx of at least $18 \mathrm{mg}$ (20 mg oxycodone hydrochloride $[\mathrm{HCl}]$ equivalent), but no more than $72 \mathrm{mg}(80 \mathrm{mg}$ oxycodone $\mathrm{HCl}$ equivalent) every $12 \mathrm{~h}$ prior to randomization. This manuscript discusses a subset of the overall population defined as those patients $\geq 65$ years old at enrollment.

\subsection{Study Design}

The enriched enrollment, randomized withdrawal (EERW) study design attributes used in the parent study were consistent with those used for other currently approved opioid analgesics [3].

A screening phase lasted up to 4 weeks, and the titration phase lasted up to 6 weeks. The purpose of the titration phase was to titrate oxycodone DETERx to a stable, effective dose that reduced the patient's pain to $\leq 4$ on the PI-NRS. The double-blind maintenance phase lasted 12 weeks, during which patients took either oxycodone DETERx or placebo twice daily. Oxycodone was taken twice daily, once in the morning and once in the evening, orally, intact, and with food. Only APAP was allowed as rescue medication ( $\leq 2000 \mathrm{mg} /$ day; $500 \mathrm{mg}$ unit doses) in both active treatment phases.

\subsection{Efficacy and Safety/Tolerability Outcome Measures}

The primary efficacy endpoint was change in the PI-NRS score from randomization baseline to Week 12 .

Secondary endpoints included improvement in pain intensity responder analysis, time to exit the study, and the Patient Global Impression of Change (PGIC) assessment.

The safety and tolerability of oxycodone DETERx were evaluated by examining common treatment-emergent adverse events (TEAEs); vital sign measurements; hematologic, biochemical, and urinalysis variables; and opioid withdrawal.

\subsection{Statistical Methods}

This post hoc analysis evaluated the study data for patients $\geq 65$ years of age. The safety population included all patients $\geq 65$ years of age who participated in the titration phase. The intent-to-treat (ITT) and randomized safety populations included all patients $\geq 65$ years who participated in the double-blind maintenance phase. The ITT population was the primary analysis population for efficacy, and the randomized safety population was the primary analysis population for safety.

The primary statistical analysis for the primary efficacy endpoint was a 2-piece linear mixed model for the PI-NRS scores from randomization baseline through Week 12 [3]. However, this model failed to converge in the subset of patients $\geq 65$ years of age, likely due to the small sample 
size; therefore, a mixed-model repeated measures analysis was used. This model included categorical fixed effects for treatment, week, and their iteration; a baseline covariate; and random subject intercepts.

A responder analysis calculated the proportions of patients demonstrating at least $30 \%$ and at least $50 \%$ improvement in pain intensity from screening baseline to Week 12 of the double-blind maintenance phase for each treatment group; patients who discontinued or had missing data were considered non-responders. Differences in the proportions between treatment groups were assessed by the Chi-square test.

The time-to-exit survival analysis was defined as the time from the randomization date to the date of early discontinuation plus 1 day. Patients who completed the study were censored at the study completion date. Kaplan-Meier estimates were derived, and the log-rank test was used to test treatment differences between the treatment groups.

For assessing change in PGIC, the number and percentage of patients (out of those with non-missing data) with each level of PGIC assessment (very much improved, improved, a little improved, no change, a little worse, worse, and very much worse) were presented by treatment group. The Cochran-Mantel-Haenszel row mean score test was used to compare the two treatment groups.

Use of APAP in the titration phase and in the doubleblind maintenance phase was calculated based on rescue medication data in an e-diary. These variables were summarized descriptively by treatment group.

Adverse events (AEs) were coded by system organ class and preferred term using the Medical Dictionary for Regulatory Activities, version 15.1 (MedDRA MSSO, McLean, VA, USA).

\section{Results}

\subsection{Patients}

A total of 88 patients $\geq 65$ years of age participated in the titration phase and were included in the safety population for this post hoc analysis. The ITT and randomized safety populations included 52 of these 88 patients, 26 each in the oxycodone DETERx and placebo groups, who participated in the randomized double-blind maintenance phase.

The median (minimum [min], maximum [max]) age was $68(65,75)$ years (Table 1$)$. The majority of patients were female $(57 \%)$, White $(69 \%)$, and not Hispanic or Latino (96\%). The median (min, max) body mass index was $29.45 \mathrm{~kg} / \mathrm{m}^{2}(18.0,49.1)$. The median (min, max) prior daily morphine-equivalent opioid dose for opioid-experienced patients was $40.5 \mathrm{mg}(27,240)$. The majority of patients required a final dose of oxycodone DETERx after
Table 1 Demographics and baseline characteristics of patients $\geq 65$ years of age (safety population)

\begin{tabular}{|c|c|}
\hline Characteristic & Total $(n=88)$ \\
\hline \multicolumn{2}{|l|}{ Age (years) } \\
\hline$n$ & 88 \\
\hline Mean (standard deviation) & $68.8(3.14)$ \\
\hline \multicolumn{2}{|l|}{ Sex, $n(\%)$} \\
\hline Male & $38(43.2)$ \\
\hline Female & $50(56.8)$ \\
\hline \multicolumn{2}{|l|}{ Race, $n(\%)$} \\
\hline Asian & $15(17.0)$ \\
\hline Black or African American & $10(11.4)$ \\
\hline White & $61(69.3)$ \\
\hline Other & $2(2.3)$ \\
\hline \multicolumn{2}{|l|}{ Ethnicity, $n(\%)$} \\
\hline Not Hispanic or Latino & $84(95.5)$ \\
\hline Hispanic or Latino & $4(4.5)$ \\
\hline \multicolumn{2}{|l|}{ Height $(\mathrm{cm})$} \\
\hline$n$ & 88 \\
\hline Mean (standard deviation) & $166.75(12.633)$ \\
\hline \multicolumn{2}{|l|}{ Weight (kg) } \\
\hline$n$ & 88 \\
\hline Mean (standard deviation) & $85.05(22.153)$ \\
\hline \multicolumn{2}{|l|}{ Body mass index $\left(\mathrm{kg} / \mathrm{m}^{2}\right)$} \\
\hline$n$ & 88 \\
\hline Mean (standard deviation) & $30.41(6.392)$ \\
\hline \multicolumn{2}{|c|}{ Prior regimen morphine equivalence (mg) } \\
\hline$n$ & 40 \\
\hline Mean (standard deviation) & $57.3(38.61)$ \\
\hline \multicolumn{2}{|c|}{ Final dose of oxycodone DETERx, $n(\%)$} \\
\hline $20 \mathrm{mg} /$ day & $12(13.6)$ \\
\hline $40 \mathrm{mg} /$ day & $26(29.5)$ \\
\hline $60 \mathrm{mg} /$ day & $21(23.9)$ \\
\hline $80 \mathrm{mg} /$ day & $14(15.9)$ \\
\hline 120 mg/day & $8(9.1)$ \\
\hline $160 \mathrm{mg} /$ day & $7(8.0)$ \\
\hline
\end{tabular}

titration of $40 \mathrm{mg} / \mathrm{day}$ (26 patients) or $60 \mathrm{mg} / \mathrm{day}$ (21 patients).

A total of 315 (48\%) patients $<65$ years of age and 36 (41\%) patients $\geq 65$ years of age discontinued the study during the titration phase (Fig. 1). The most common reasons for discontinuation among both of these groups were not meeting the entrance criteria for the double-blind maintenance phase (19\% of patients $<65$ years of age and $16 \%$ of patients $\geq 65$ years of age) and AEs (13\% of patients $<65$ and $\geq 65$ years of age).

Among patients $\geq 65$ years of age, $8(31 \%)$ patients in the oxycodone DETERx group and $13(50 \%)$ patients in the placebo group discontinued during the double-blind maintenance phase. In the oxycodone DETERx group, the 
Fig. 1 Patient disposition

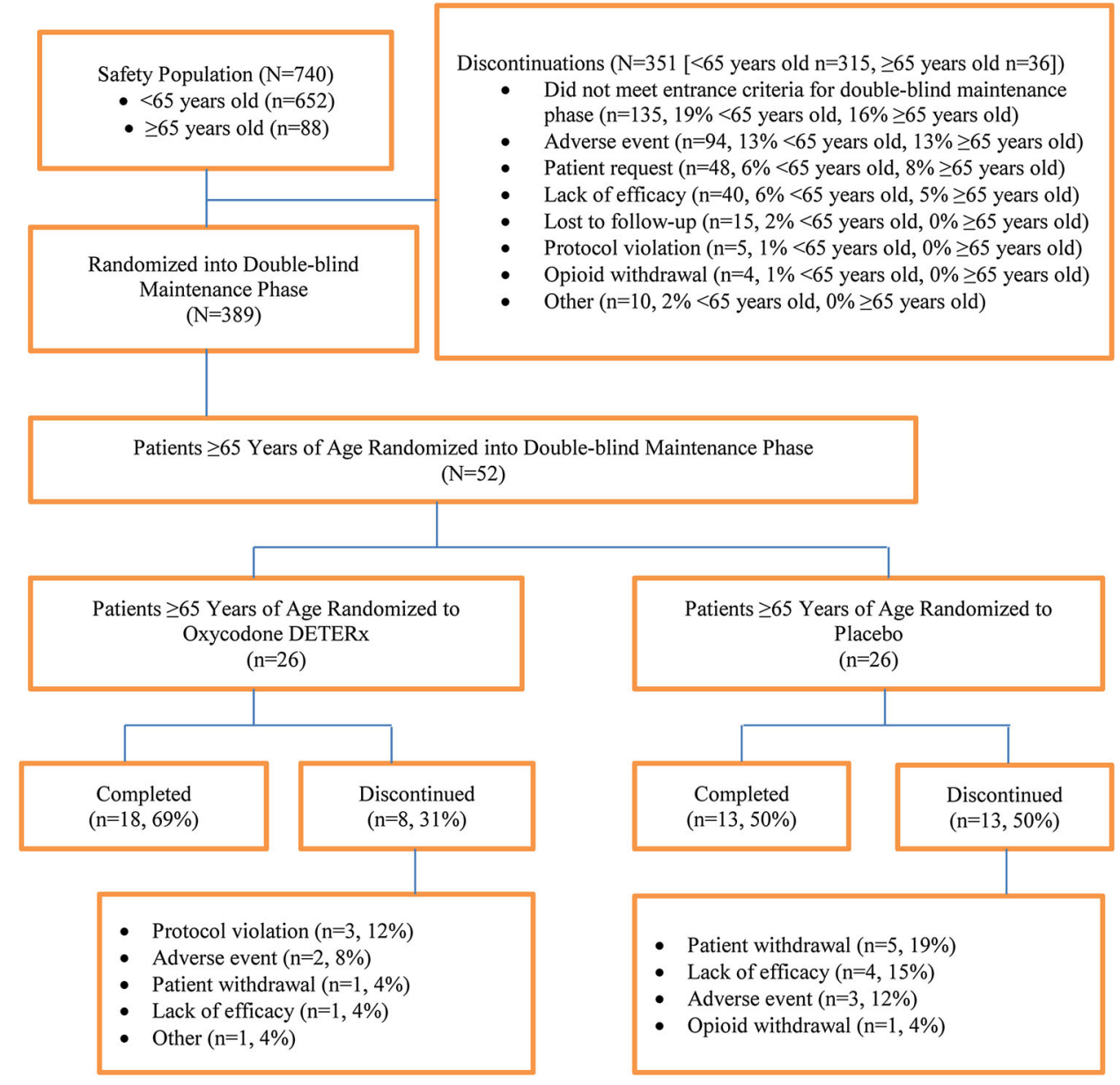

most common reasons for discontinuation were protocol violations (3 patients, 12\%) and AEs (2 patients, $8 \%$ ). In the placebo group, the most common reasons for discontinuation were patient withdrawal (5 patients, 19\%) and lack of efficacy (4 patients, 15\%).

\subsection{Efficacy}

Weekly pain intensity scores from screening through Week 12 of the double-blind maintenance phase were similar among patients $\geq 65$ years of age and patients $<65$ years of age (Fig. 2).

Mean changes in PI-NRS score from screening baseline to double-blind randomization baseline were clinically and statistically significant in patients $<65$ years of age and patients $\geq 65$ years of age $(p<0.0001)$. Among patients $<65$ years of age, the least squares (LS) mean [standard error (SE)] change from screening baseline to randomization baseline was $-3.88(0.130)$ among patients subsequently randomized to oxycodone DETERx and -3.98 (0.128) among patients subsequently randomized to placebo. Similarly, among patients $\geq 65$ years of age, the LS mean (SE) change from screening baseline was -4.29

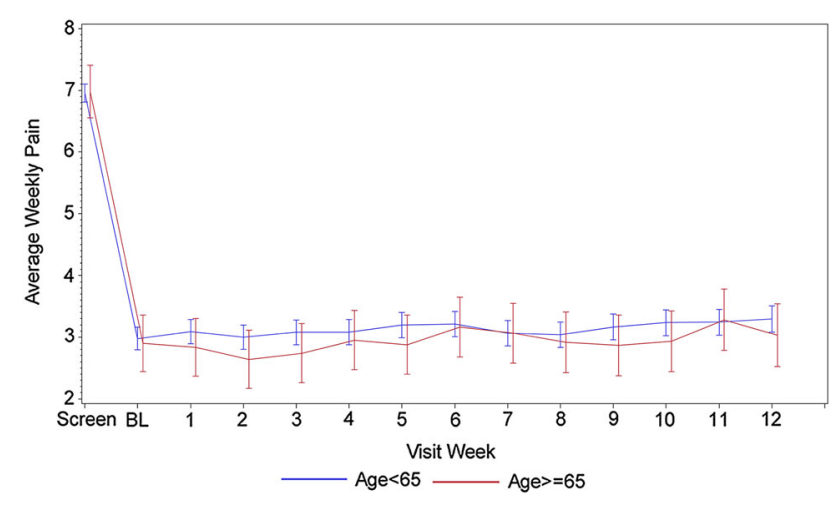

Fig. 2 Weekly pain intensity (measured using an 11-point (0-10) numerical rating scale) scores for patients taking oxycodone DETERx by age (intent-to-treat population)

(0.289) in the prospective oxycodone DETERx group and $-3.96(0.290)$ in the prospective placebo group.

During the double-blind maintenance phase, patients $<65$ years of age in the oxycodone DETERx group experienced a significant decrease in mean pain score compared with patients $<65$ years of age in the placebo group, with a treatment difference $[95 \%$ confidence interval (CI)] of $-0.8(-1.2$ to -0.4$)$ (Table 2). Among 
Table 2 Change in pain score by age during the double-blind maintenance phase (intent-to-treat population)

\begin{tabular}{|c|c|c|c|c|}
\hline \multirow[t]{2}{*}{ Statistic } & \multicolumn{2}{|l|}{ Age $<65$ years } & \multicolumn{2}{|l|}{ Age $\geq 65$ years } \\
\hline & $\begin{array}{l}\text { Oxycodone } \\
\text { DETERx }(n=164)\end{array}$ & $\begin{array}{l}\text { Placebo } \\
(n=168)\end{array}$ & $\begin{array}{l}\text { Oxycodone } \\
\text { DETERx }(n=26)\end{array}$ & $\begin{array}{l}\text { Placebo } \\
(n=26)\end{array}$ \\
\hline Mean change in average pain score (standard error) & $0.6(0.2)$ & $1.4(0.2)$ & $0.4(0.3)$ & $0.9(0.5)$ \\
\hline Treatment difference ( $95 \%$ confidence interval) & $-0.8(-1.2$ to -0.4$)$ & & $-0.9(-2.1$ to 0.3$)$ & \\
\hline$p$ value & 0.0004 & & 0.12 & \\
\hline
\end{tabular}

patients $\geq 65$ years of age, the treatment difference (95\% CI) between the oxycodone DETERx group versus the placebo group $[-0.9(-2.1$ to 0.3$)]$ was greater than that for patients $<65$ years old; however, the difference was not statistically significant due to the small sample size.

The responder analysis showed that among patients $\geq 65$ years of age, the oxycodone DETERx group had a significantly greater proportion of patients $(62 \%)$ with $\geq 30 \%$ reduction in pain intensity at Week 12 compared with the placebo group $(27 \%)(p=0.0128$; Fig. 3). Among patients $<65$ years old, the difference between the oxycodone DETERx group and placebo group was also statistically significant, with $47 \%$ of patients in the oxycodone DETERx group and $34 \%$ of patients in the placebo group reporting a $\geq 30 \%$ reduction in pain intensity at Week 12 ( $p=0.0139)$.

The proportion of patients with $\geq 50 \%$ reduction in pain intensity at Week 12 was also significantly greater in the oxycodone DETERx group (54\%) than in the placebo group (27\%) among patients $\geq 65$ years of age $(p=0.0501)$. Results were similar for patients $<65$ years of age, showing statistically significantly greater proportions of patients with $\geq 50 \%$ reductions in pain intensity in the oxycodone DETERx group $(36 \%)$ than in the placebo group $(24 \%)$ at Week $12(p=0.0181)$.

Among patients in the oxycodone DETERx group who had data at Week 12 or the final study visit, the

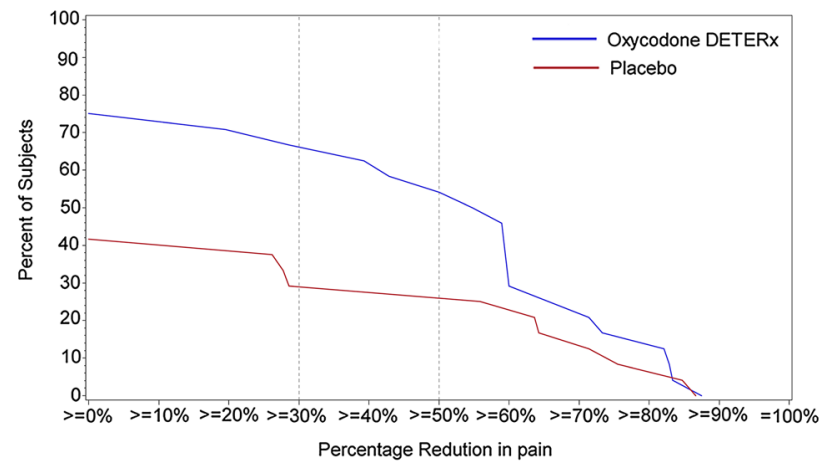

Fig. 3 Responder analysis for pain intensity percent reduction at Week 12 in patients $\geq 65$ years old (intent-to-treat population) proportions of patients who reported being 'very much improved', 'improved', and 'a little improved' were generally similar among patients $<65$ years old and patients $\geq 65$ year old, although the $\geq 65$-year-old had higher proportions of patients in the categories of "very much improved' and 'improved' (Table 3). At the final study visit, $68 \%$ of patients $<65$ years of age and $81 \%$ of patients $\geq 65$ years of age reported being 'very much improved' or 'improved'.

During the double-blind maintenance phase, the proportion of patients who remained in the study was higher in the oxycodone DETERx group than in the placebo group starting at about Week 4, although among patients $\geq 65$ years of age, the difference between the two groups at the time to exit the study was not statistically significant ( $p=0.1113$ ) due to the small sample size (Fig. 4). Similar results for time to exit were observed for patients $<65$ years of age; the difference between the oxycodone DETERx group and placebo group among these patients was statistically significant $(p=0.0280)$.

Only APAP was allowed as rescue medication, and overall use was low. Among patients $\geq 65$ years of age, the mean (SD) number of doses per week of rescue medication for breakthrough pain during the titration phase was 2.1 (2.74), and the mean (SD) dosage per week was $1677 \mathrm{mg}$ (2434.9). Among patients $<65$ years of age, the mean (SD) number of doses per week of rescue medication for breakthrough pain during the titration phase was 2.5 (3.64), and the mean (SD) dosage per week was $2173 \mathrm{mg}$ (3263.5).

During the double-blind maintenance phase, rescue medication use among patients $<65$ and $\geq 65$ years of age in the oxycodone DETERx group was similar, and in both age groups, patients in the oxycodone DETERx group required less rescue medication for breakthrough pain than patients in the placebo group. The mean (SD) number of doses per week of rescue medication among patients $\geq 65$ years of age in the oxycodone DETERx group was 1.0 (1.89), compared with 1.8 (2.57) for patients in this age group in the placebo group, and the mean (SD) dosage per week was $1055 \mathrm{mg}$ (2162.0) in the oxycodone DETERx group compared with $1596 \mathrm{mg}$ (2395.7) for patients in this age group in the placebo group. 
Table 3 Patient global impression of change by age (intent-to-treat population)

\begin{tabular}{lll}
\hline $\begin{array}{l}\text { Visit } \\
\text { Patient Global Impression of Change }\end{array}$ & $\begin{array}{l}\text { Oxycodone DETERx } \\
\text { Age }<65 \text { years }(n=167)\end{array}$ & $\begin{array}{l}\text { Oxycodone DETERx } \\
\text { Age } \geq 65 \text { years }(n=88)\end{array}$ \\
\hline Week 12, $n(\%)$ & $n=104$ & $n=18$ \\
Very much improved & $30(28.8)$ & $6(33.3)$ \\
Improved & $43(41.3)$ & $9(50.0)$ \\
A little improved & $20(19.2)$ & $3(16.7)$ \\
No change & $9(8.7)$ & 0 \\
A little worse & 0 & 0 \\
Worse & $2(1.9)$ & 0 \\
Very much worse & 0 & 0 \\
Final study visit ${ }^{\mathrm{a}}, n(\%)$ & $n=158$ & $n=26$ \\
Very much improved & $43(27.2)$ & $8(30.8)$ \\
Improved & $65(41.1)$ & $13(50.0)$ \\
A little improved & $29(18.4)$ & $3(11.5)$ \\
No change & $14(8.9)$ & $1(3.8)$ \\
A little worse & $3(1.9)$ & 0 \\
Worse & $4(2.5)$ & $1(3.8)$ \\
Very much worse & 0 & 0
\end{tabular}

a The final visit is the last non-missing visit after randomization baseline

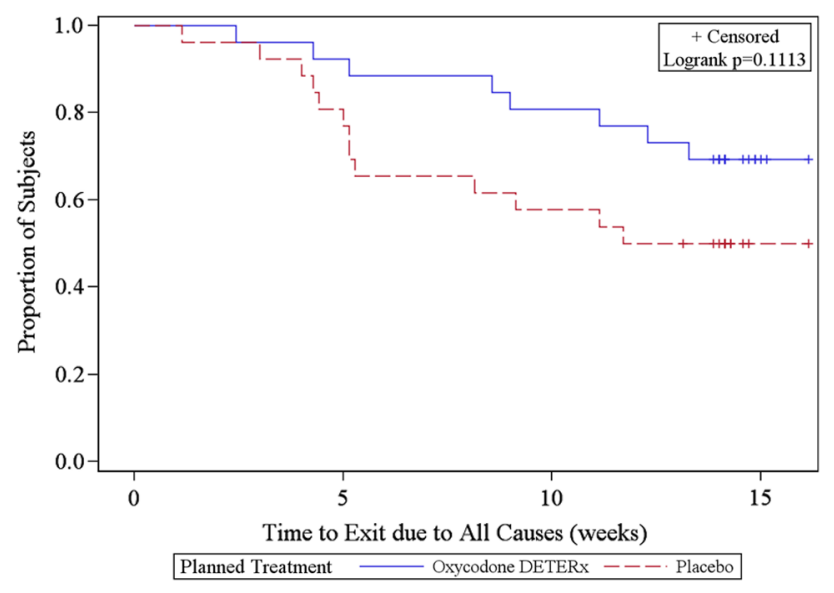

Fig. 4 Time to exit from the study using the Kaplan-Meier method-patients $\geq 65$ years of age

Most patients randomized to oxycodone DETERx in the double-blind maintenance phase required no adjustments in dose. Among patients $\geq 65$ years of age, one opioid-naïve patient at $60 \mathrm{mg} /$ day oxycodone DETERx required a down titration; no adjustment was required for the remaining 11 opioid-naïve patients. Among the 14 opioid-experienced patients $\geq 65$ years of age, one patient at $80 \mathrm{mg} /$ day oxycodone DETERx required a down titration and an up titration. Among patients $<65$ years of age, only one patient, an opioid-experienced patient at $120 \mathrm{mg} /$ day oxycodone DETERx, required a down titration.

\subsection{Safety}

Only minor fluctuations in mean vital sign measurements from the double-blind randomization baseline to the final study visit were noted in the oxycodone DETERx group. Changes in mean vital sign measurements were similar among patients $<65$ years of age and patients $\geq 65$ years of age. No changes from baseline were clinically significant.

During the titration phase, similar proportions of subjects $<65$ years of age and $\geq 65$ years of age experienced at least one TEAE, serious TEAE, treatment-related TEAE, and TEAE leading to treatment termination (Table 4). One death was reported among patients $<65$ years of age, but was not related to oxycodone DETERx.

During the titration phase, proportions of patients experiencing nausea, constipation, headache, and somnolence were generally similar among patients $\geq 65$ years of age and patients $<65$ years of age. Greater proportions of patients $\geq 65$ years of age experienced pruritus, dizziness, dry mouth, upper respiratory tract infection, and tremor compared with patients $<65$ years of age. A greater proportion of subjects $<65$ years of age experienced vomiting.

During the double-blind maintenance phase, the proportions of subjects $<65$ years of age and $\geq 65$ years of age who experienced at least one TEAE, serious TEAE, and treatment-related TEAE were similar (Table 5).

During the double-blind maintenance phase, the most frequently reported ( $\geq 5 \%$ of patients in either treatment group) preferred terms of TEAEs among patients 
Table 4 Summary of treatment-emergent adverse events by age during the titration phase (safety population)

\begin{tabular}{lll}
\hline & $\begin{array}{l}\text { Oxycodone DETERx } \\
<65 \text { years of age }(n=652) \\
n(\%)\end{array}$ & $\begin{array}{l}\text { Oxycodone DETERx } \\
\geq 65 \text { years of age }(n=88) \\
n(\%)\end{array}$ \\
\hline Number of TEAEs & 1032 & 165 \\
Patients with TEAEs & $411(63.0)$ & $51(58.0)$ \\
Patients with serious TEAEs & $7(1.1)$ & $1(1.1)$ \\
Patients with treatment-related TEAEs & $321(49.2)$ & $45(51.1)$ \\
Patients with treatment-related serious/severe TEAEs & $2(0.3)$ & 0 \\
Patients with TEAEs leading to study treatment termination & $93(14.3)$ & $12(13.6)$ \\
Patients with TEAEs leading to death & $1(0.2)^{\mathrm{a}}$ & 0 \\
\hline
\end{tabular}

TEAEs treatment-emergent adverse events

${ }^{\text {a }}$ Not related to oxycodone DETERx per investigator assessment

Table 5 Summary of treatment-emergent adverse events by age during the double-blind maintenance phase (randomized safety population)

\begin{tabular}{lll}
\hline & $\begin{array}{l}\text { Oxycodone DETERx } \\
<65 \text { years of age }(n=167) \\
n(\%)\end{array}$ & $\begin{array}{l}\text { Oxycodone DETERx } \\
\geq 65 \text { years of age }(n=26) \\
n(\%)\end{array}$ \\
\hline Number of TEAEs & 243 & 50 \\
Patients with TEAEs & $108(64.7)$ & $17(65.4)$ \\
Patients with serious TEAEs & $2(1.2)$ & 0 \\
Patients with treatment-related TEAEs & $56(33.5)$ & $9(34.6)$ \\
Patients with treatment-related serious/severe TEAEs & 0 & 0 \\
Patients with TEAEs leading to study treatment termination & $14(8.4)$ & $1(3.8)$ \\
Patients with TEAEs leading to death & 0 & 0
\end{tabular}

TEAEs treatment-emergent adverse events

$\geq 65$ years of age were nausea and diarrhea (each $15.4 \%$ of patients), followed by constipation, dizziness, nasopharyngitis, rash, tremor, and urinary tract infection (each $7.7 \%$ of patients). Among patients $<65$ years of age, nausea $(10.2 \%)$ and headache $(6.6 \%)$ were the most frequently reported preferred terms of TEAEs during the double-blind maintenance phase.

Clinical laboratory test results, vital sign measurements, and opioid withdrawal symptoms for patients $\geq 65$ years old were not different from those for patients $<65$ years old.

\section{Discussion}

Effectively treating severe pain in patients is a clinical challenge often requiring a multimodal approach using pharmacological (e.g., analgesics from one or a combination of drug classes) and non-pharmacological approaches (e.g., cognitive behavioral therapy, physical therapy). In the patient population $\geq 65$ years of age, clinicians must also address the fact that many patients are frail and have comorbid conditions. As patients age, morbidity, mortality, and social issues increase more rapidly, putting a strain on the patient's support network as well as on healthcare providers and the system [16], which is one reason appropriately treating chronic pain in the elderly is important. Epidemiological studies have shown that painrelated issues are present in $25-50 \%$ of older people residing in the community and in $30-49 \%$ of elders resident in nursing homes [17-19]. Untreated pain in the elderly can lead to adverse outcomes such as increased risk of suicide (odds ratio 7.52; 95\% CI 4.93-11.46 in cases of severe pain in the elderly), functional impairment, gait impairment, falls, delayed rehabilitation, decreased recreational/social activities, mood changes (e.g., higher rates of depression and anxiety), decreased socialization, sleep and appetite abnormalities, and increased alcohol consumption, all of which culminate in decreased quality of life for the patient and for those in the patient's support structure [17, 19-23].

The American Geriatrics Society Panel on Pharmacological Management of Persistent Pain in Older Persons prepared a guideline in 2009 specifically addressing the 
need for appropriate pharmacological management of older patients with chronic pain [24]. Among these recommendations was the use of opioid analgesics in properly selected and monitored patients [25-30]. Moreover, in this patient population, clinicians must consider the evidence indicating that the use of nonsteroidal anti-inflammatory analgesics and cyclo-oxygenase- 2 inhibitors may result in insidious onset of serious and potentially life-threatening gastrointestinal and cardiovascular AEs [31], which has led to opioids as an important treatment option for physicians in the management of pain in the elderly. As with any analgesic, there are potential risks associated with opioids, which include nausea, vomiting, constipation, sedation, respiratory depression, overdose, abuse, addiction, and death [32].

Of increasing concern in this patient population is appropriate treatment of pain. Pain occurs in $80 \%$ of elderly patients with cancer, but these patients are less likely to receive adequate analgesia than younger patients with cancer [33-35]. A review of $>21,000$ elderly nursing home residents showed that $25 \%$ of residents with pain did not receive any analgesic medication [19]. Another study showed similar results; elderly patients received fewer opioids than younger patients [36]. Reasons for under-utilization of opioids in elderly patients may include poor pain assessment (partly attributable to communication issues resulting from dysphagia, hearing impairment, education, culture, or dementia), fear of polypharmacy, opiophobia (including concerns about tolerance, physical dependence, addiction, and AEs) [37], and known potential risks of opioids.

Limited data exist on the effects of oxycodone treatment regimens in elderly patients and on the challenges posed by currently marketed ER formulations in this patient population. Meeting the challenges of providing effective treatment for chronic pain in the elderly is a public health concern, as the elderly population is increasing and the elderly experience more pain conditions than younger adults or children [7]. Many currently available oral ER opioids have limitations in elderly patients; they can be difficult to swallow [38-40], leading patients and their caregivers to tamper with and damage the intact formulations to overcome this difficulty [5, 7]. Hard, monolithic tablet formulations have been associated with post-marketing reports of choking, gagging, regurgitation, and tablets stuck in the throat $[3,38,39]$. Chewing, crushing, cutting, or dissolving these tablets to facilitate swallowing can cause rapid release and absorption of a potentially fatal dose of opiate. In other cases when tampering occurs with an agonist/antagonist combination product, release of the antagonist could lead to exacerbation of pain and/or withdrawal symptoms [38-42]. Oxycodone DETERx can provide a useful option for patients with dysphagia, as the capsule contents may be sprinkled onto soft foods or into a cup and then directly administered into the mouth. The capsules are relatively easy to open, offering these alternate dosing options to elderly patients with decreased manual dexterity due to osteoarthritis or other dexterity-limiting conditions. In addition, in vitro studies have shown that oxycodone DETERx may be administered directly through enteral tubes without changing the release properties [14]. Studies conducted to assess the abuse deterrence characteristics demonstrated that administration of oxycodone DETERx either crushed or chewed did not increase the maximum drug concentration $\left(C_{\max }\right)$ or total exposure (AUC) relative to dosing the drug intact as a capsule, indicating that crushing or chewing does not compromise its ER properties [12].

The treatment difference of the analgesic effect of oxycodone DETERx in patients $\geq 65$ years of age was similar numerically to that of patients $<65$ years of age, indicating that oxycodone DETERx is effective in the management of pain attributable to CLBP in the elderly, although the result was not statistically significant. The lack of statistical significance of the primary endpoint comparison in the $\geq 65$-year-old cohort is a result of the small sample size; given the resultant effect size for this age cohort, there would be only $36 \%$ power to detect a difference in effectiveness with the number of subjects $\geq 65$ years of age in this study [43]. Improved pain control was also supported in that throughout the 12-week doubleblind maintenance phase, the majority of patients did not require a dose adjustment once they achieved a stable dose of oxycodone DETERx, despite only being allowed to mitigate breakthrough pain with APAP. In addition, the dropout rate attributable to lack of effectiveness was low.

Results for secondary endpoints, including for the responder analysis, PGIC, and time-to-exit analyses, were consistent for patients $<65$ years of age and patients $\geq 65$ years of age.

In both the titration and double-blind maintenance phases, the incidence of AEs, serious adverse events (SAEs), treatment-related AEs, and treatment-related SAEs were similar between patients $<65$ years and patients $\geq 65$ years of age, indicating that oxycodone DETERx may offer geriatricians treating chronic pain another abuse-deterrent opioid option for older patients who have dysphagia or odynophagia (painful swallowing). The AE profile for oxycodone DETERx was consistent with other ER opioid formulations and was similar between patients $<65$ years of age and patients $\geq 65$ years of age.

As with any clinical study, there are limitations. The sample size for the cohort of patients $\geq 65$ years of age was small, with only 26 patients each in the active and placebo groups, which limited the ability to detect statistically significant differences between the two groups. However, 
the sample size for the age cohort was not atypical and was similar to other studies [40, 44]. The clinical study design was an EERW design of a phase III study adherent to the requirements of the FDA for a chronic pain indication, which may limit the generalizability of the results to an elderly, dysphagic patient population. The treatment in this study was oxycodone DETERx unimodal therapy; use of a more real-world treatment paradigm of multi-modal therapy was not possible in order to avoid confounding of the study results.

\section{Conclusions}

Oxycodone DETERx is an easy-to-administer, abuse-deterrent alternative to hard, monolithic tablet formulations of ER opioids that may be difficult to swallow, particularly in the elderly. This analysis showed that oxycodone DETERx was generally efficacious (i.e., clinically relevant), although not statistically significant due to the relatively small sample size, and well tolerated in the $\geq 65$ year-old subgroup of the study population, with no newly identified (i.e., AEs currently not already known based on the pharmacology of opioids) safety concerns.

Acknowledgements Editorial support for writing this manuscript was provided by Teresa Faulkner, MA, of Rho, Inc.

\section{Compliance with Ethical Standards}

Conflict of interest Authors Melinda O'Connor and Scott Lagasse are salaried (including stock) employees of Collegium Pharmaceutical, Inc., the company that funded the study and the writing of this manuscript. At the time this manuscript was written, author Ernest A. Kopecky was a salaried (including stock) employee of Collegium Pharmaceutical, Inc. Ben Vaughn is a salaried employee of Rho, Inc., a contract research organization that performed the statistical analyses for the manuscript. Mr. Vaughn has no personal financial ties to Collegium Pharmaceutical, Inc. and no ownership in any form in Rho, Inc.

Funding The study was funded by Collegium Pharmaceutical, Inc.

Ethical approval and informed consent The study was conducted according to Good Clinical Practice guidelines and the Declaration of Helsinki. The clinical protocol for the parent study was reviewed and approved by an institutional review board (Quorum Review IRB, Seattle, WA, USA). All patients provided written consent to participate in the parent study before study-related procedures or assessments were conducted.

Open Access This article is distributed under the terms of the Creative Commons Attribution-NonCommercial 4.0 International License (http://creativecommons.org/licenses/by-nc/4.0/), which permits any noncommercial use, distribution, and reproduction in any medium, provided you give appropriate credit to the original author(s) and the source, provide a link to the Creative Commons license, and indicate if changes were made.

\section{References}

1. Global Industry Analysts, Inc. Report; 2011. http://www.prweb. com/pdfdownload/8052240.pdf. Accessed 6 June 2017.

2. Newsroom Archive. 2010 census shows 65 and older population growing faster than total U.S. population. https://www.census. gov/newsroom/releases/archives/2010_census/cb11-cn192.html. Accessed 6 June 2017.

3. Katz N, Kopecky EA, OConnor M, et al. A phase 3, multicenter, randomized, double-blind, placebo-controlled, safety, tolerability, and efficacy study of Xtampza ER in patients with moderateto-severe chronic low back pain. Pain. 2015;156(12):2458-67.

4. Maxwell JC. The prescription drug epidemic in the United States: a perfect storm. Drug Alcohol Rev. 2011;30(3):264-70.

5. Pergolizzi JV Jr, Taylor R Jr, Nalamachu S, et al. Challenges of treating patients with chronic pain with dysphagia (CPD): physician and patient perspectives. Curr Med Res Opin. 2014;30(2):191-202.

6. Center for Drug Evaluation and Research (CDER). Guidance for industry: size, shape, and other physical attributes of generic tablets and capsules. Rockville: U.S. Department of Health and Human Services, Food and Drug Administration, Center for Drug Evaluation and Research; 2015.

7. Argoff CE, Kopecky EA. Patients with chronic pain and dysphagia (CPD): unmet medical needs and pharmacologic treatment options. Curr Med Res Opin. 2014;30(12):2543-59.

8. Coluzzi PH, Fairbairn BS. The management of pain in terminally ill cancer patients with difficulty swallowing. Am J Hosp Palliat Med. 1999;16(6):731-7.

9. Zorba Paster R. Chronic pain management issues in the primary care setting and the utility of long-acting opioids. Expert Opin Pharmacother. 2010;11(11):1823-33.

10. Gudin JA. Assessment of extended-release opioid analgesics for the treatment of chronic pain. J Pain Palliat Care Pharmacother. 2013;27(1):49-61.

11. Raffa RB, Burmeister JJ, Yuvasheva E, et al. QTc interval prolongation by D-propoxyphene: what about other analgesics? Expert Opin Pharmacother. 2012;13(10):1397-409.

12. Kopecky EA, Fleming AB, Noonan PK, et al. Impact of physical manipulation on in vitro and in vivo release profiles of oxycodone DETERx ${ }^{\circledR}$ : an extended-release, abuse-deterrent formulation. J Opioid Manag. 2014;10(4):233-46.

13. Kopecky E, Fleming A, Findlen K, et al. Oxycodone DETERx, an extended-release, abuse-deterrent formulation for management of patients with chronic pain and chronic pain with dysphagia. J Pain. 2014;15(4):S90.

14. Fleming AB, Carlson DR, Varanasi RK, et al. Evaluation of an extended-release, abuse-deterrent, microsphere-in-capsule analgesic for the management of patients with chronic pain with dysphagia (CPD). Pain Pract. 2015;16(3):334-44.

15. Katz N. Enriched enrollment randomized withdrawal trial designs of analgesics: focus on methodology. Clin $J$ Pain. 2009;25(9):797-807.

16. Ferrell B, Ferrell BA. Pain in the elderly: a report of the Task Force on Pain in the Elderly of the International Association for the Study of Pain. Seattle: IASP Press; 1996.

17. Chodosh J, Solomon DH, Roth CP, et al. The quality of medical care provided to vulnerable older patients with chronic pain. J Am Geriatr Soc. 2004;52(5):756-61.

18. McClean WJ, Higginbotham NH. Prevalence of pain among nursing home residents in rural New South Wales. Med J Aust. 2002;177(1):17-20.

19. Won AB, Lapane KL, Vallow S, et al. Persistent nonmalignant pain and analgesic prescribing patterns in elderly nursing home residents. J Am Geriatr Soc. 2004;52(6):867-74. 
20. AGS Panel on Persistent Pain in Older Persons. The management of persistent pain in older persons. J Am Geriatr Soc. 2002;50(S6):205-24.

21. Juurlink DN, Herrmann N, Szalai JP, et al. Medical illness and the risk of suicide in the elderly. Arch Intern Med. 2004;164(11):1179-84.

22. Jakobsson U, Rahm Hallberg I, Westergren A. Pain management in elderly persons who require assistance with activities of daily living: a comparison of those living at home with those in special accommodations. Eur J Pain. 2004;8(4):335-44.

23. Bosley BN, Weiner DK, Rudy TE, et al. Is chronic nonmalignant pain associated with decreased appetite in older adults? Preliminary evidence. J Am Geriatr Soc. 2004;52(2):247-51.

24. Ferrell B, Argoff CE, Epplin J, et al. Pharmacological management of persistent pain in older persons. J Am Geriatr Soc. 2009;57(8):1331-46. doi:10.1111/j.1532-5415.2009.02376.x.

25. Avouac J, Gossec L, Dougados M. Efficacy and safety of opioids for osteoarthritis: a meta-analysis of randomized controlled trials. Osteoarthr Cartil. 2007;15(8):957-65.

26. Chou R, Qaseem A, Snow V, et al. Diagnosis and treatment of low back pain: a joint clinical practice guideline from the American College of Physicians and the American Pain Society. Ann Intern Med. 2007;147(7):478-91.

27. Eisenberg E, McNicol ED, Carr DB. Efficacy and safety of opioid agonists in the treatment of neuropathic pain of nonmalignant origin. J Am Med Assoc. 2005;293(24):3043-52.

28. Moulin DE, Clark AJ, Gilron I, et al. Pharmacological management of chronic neuropathic pain-consensus statement and guidelines from the Canadian Pain Society. Pain Res Manag. 2007;12(1):13-21.

29. Nicholson B, Passik SD. Management of chronic noncancer pain in the primary care setting. South Med J. 2007;100(10):1028-36.

30. Trescot AM, Helm S, Hansen H, et al. Opioids in the management of chronic non-cancer pain: an update of American Society of the Interventional Pain Physicians' (ASIPP) Guidelines. Pain Physician. 2008;11(2 Suppl):S5-62.

31. Singh $\mathrm{G}, \mathrm{Wu} \mathrm{O}$, Langhorne $\mathrm{P}$, et al. Risk of acute myocardial infarction with nonselective non-steroidal anti-inflammatory drugs: a meta-analysis. Arthritis Res Ther. 2006;8:R153.
32. Benyamin R, Trescot AM, Datta S, Buenaventura R, Adlaka R, Sehgal N, et al. Opioid complications and side effects. Pain Physician. 2008;11(2 Suppl):S105-20.

33. Rao A, Cohen HJ. Symptom management in the elderly cancer patient: fatigue, pain, and depression. J Natl Cancer Inst Monogr. 2004;2004(32):150-7.

34. Cleary JF, Carbone PP. Palliative medicine in the elderly. Cancer. 1997;80(7):1335-47.

35. Gloth FM 3rd. Pain management in older adults: prevention and treatment. J Am Geriatr Soc. 2001;49(2):188-99.

36. Viganó A, Bruera E, Suarez-Almazor ME. Age, pain intensity, and opioid dose in patients with advanced cancer. Cancer. 1998;83(6):1244-50.

37. Auret K, Schug SA. Underutilisation of opioids in elderly patients with chronic pain. Drugs Aging. 2005;22(8):641-54.

38. OxyContin [package insert] Purdue Pharma L.P., Stamford, CT, 2007. http://www.accessdata.fda.gov/drugsatfda_docs/label/2008/ 020553s059lbl.pdf. Accessed 6 June 2017.

39. Opana ER [package insert] Endo Pharmaceuticals Inc., Chadds Ford, PA. 2012. http://www.accessdata.fda.gov/drugsatfda_docs/ label/2013/201655s004lbl.pdf. Accessed 6 June 2017.

40. Hysingla ER [package insert] Purdue Pharma L.P., Stamford, CT. 2014. http://www.accessdata.fda.gov/drugsatfda_docs/label/ 2014/206627s000lbl.pdf. Accessed 6 June 2017.

41. Embeda [medication guide] Pfizer Inc., New York, NY. 2014. http://www.fda.gov/downloads/Drugs/DrugSafety/UCM179172. pdf. Accessed 6 June 2017.

42. Troxyca ER [medication guide] Pfizer, Inc., New York, NY. 2016. http://www.accessdata.fda.gov/drugsatfda_docs/label/ 2016/207621s004lbl.pdf\#page=32. Accessed 6 June 2017.

43. SAS Software, Version 9.4 of the SAS System for Windows. Copyright (C) 2014 SAS Institute Inc.

44. Zohydro ER [medical review] Zogenix Inc., Emeryville, CA. 2013. http://www.accessdata.fda.gov/drugsatfda_docs/nda/2013/ 202880Orig1s000MedR.pdf. Accessed 6 June 2017. 